\author{
PRESIDENT \\ VICE-PRESIDENT \\ PAST PRESIDENT \\ ORGANIZING COMMITTEE
}

\author{
Andreas Quirrenbach \\ David R. Silva \\ Rolf-Peter Kudritzki \\ Michael G. Burton \\ Xiangqun Cui \\ Ian S. McLean \\ Eugene F. Milone \\ Jayant Murthy \\ Stephen T. Ridgway \\ Gražina Tautvaišiene \\ Andrei A. Tokovinin \\ Guillermo Torres
}

\title{
PARTICIPATING COMMISSIONS
}

Commission 21

Commission 25

Commission 30

Commission 54
Galactic and Extragalactic Background Radiation Stellar Photometry \& Polarimetry

Radial Velocities

Optical \& Infrared Interferometry

\section{DIVISION WORKING GROUPS}

Division IX WG

Site Testing Instruments

Division IX WG

Sky Surveys

INTER DIVISION WORKING GROUPS

Division IX-X WG Encouraging the International Development of Antarctic Astronomy

Inter-Div. IX-X-XI WG Astronomy from the Moon

PROCEEDINGS BUSINESS SESSION, 14 August 2009

\section{Introduction}

Division IX provides a forum for astronomers engaged in the planning, development, construction, and calibration of optical and infrared telescopes and instrumentation, as well as observational procedures including data processing. A few years ago, discussions were started about changes in the structure of Division IX, with the aim of bringing it more in line with today's world of large coordinated projects and multi-national observatories. The course of this process, and further steps to be taken in the period from 2009 to 2012, were at the focus of the deliberations at the business meeting of Division IX at the IAU General Assembly in Rio de Janeiro. 


\section{Re-Structuring of Division IX}

The introduction of new directions for Division IX started with the creation of a Working Group on Optical and Infrared Interferometry, which meanwhile has become Commission 54. In preparation for the General Assembly, this commission has informed a large number of astronomers active in optical/infrared interferometry about the commission's activities, and encouraged them to become members of the IAU (if they weren't already) and of Commission 54.

At the General Assembly, the wish of Commission 21 to move from Division III to Division IX, and to change its name to "Galactic and Extragalactic Background Radiation" was approved by the Executive Committee. These changes reflect the current interests and research activities of the majority of the members of the commission, and should help the commission to attract new members.

Several important topics that should fall under the purview of Division IX are currently not covered by the commissions and working groups of the division. It is therefore foreseen to establish new working groups on large telescopes, on medium-size and small telescopes, and on adaptive optics, respectively.

An open question concerns the role of optical and infrared astronomy from space within Division IX. Discussions with the relevant community and with Division XI will be needed to define the appropriate distribution of tasks between the two divisions.

\section{Support of the Instrumentation Community by Division IX}

Among the important tasks of Division IX are facilitating professional contacts between astronomers interested in optical and infrared instrumentation, and representing the interests of this group within the wider astronomical community. It was noted that the IAU sponsors relatively few symposia with a focus on instrumentation. The largest conferences on astronomical instrumentation are organized on a bi-annual basis by SPIE. These serve many IAU members well, but for others the format is not ideal, and for some the cost of attending may be prohibitively high. Division IX should thus try to prepare suggestions how the IAU could take a stronger role in the organization and sponsorship of instrumentation-related conferences.

Many astronomers engaged in the development of state-of-the-art instrumentation spend a large fraction of their time on the design and construction of instruments, and on managing their teams. Completed instruments are frequently handed over to observatories, giving the original builders only a limited role in their scientific exploitation. As a consequence, the accomplishments of instrument builders are in many cases not reflected in their publication records, which can potentially harm their prospects in hiring and review processes. It was proposed that Division IX should assess this situation, and propose improvements if appropriate.

Andreas Quirrenbach President of the Division 